(c) 1980. The Genetical Society of Great Britain

\title{
COMBINED GENETIC AND CYTOLOGICAL ANALYSIS OF POSITIVE AND NEGATIVE INTERFERENCE IN AN INTERCHANGE HETEROZYGOTE OF RYE (SECALE CEREALE L.)*
}

\author{
J. SYBENGA and I. MASTENBROEK \\ Department of Genetics, Agricultural University, Wageningen
}

Received 11.vii.79

\section{Summary}

Segregation of a translocation breakpoint and two marker genes, located in different non-translocation arms, showed strong negative interference between the segments bordered by the markers and the transiocation. Cytological data indicated a similarly strong negative interference between the interstitial segments, which make up part of the segments analysed genetically. Since coincidence is stronger than compatible with random anaphase II segregation of chromatids, it is suggested that the relatively proximal chiasmata involved in recombination in the segments analysed, cause chromatid pre-orientation, leading to preferential A II segregation.

\section{InTRODUCTION}

THE pattern of interference in the multivalent of interchange heterozygotes deviates significantly from that in normal chromosomes. Within individual segments interference is normal (positive, except occasionally in restricted areas around the centromere where it can be negative, cf. Newcombe, 1941). Between the two translocated segments ( $R$ and $S$ in fig. 1 ) and between the

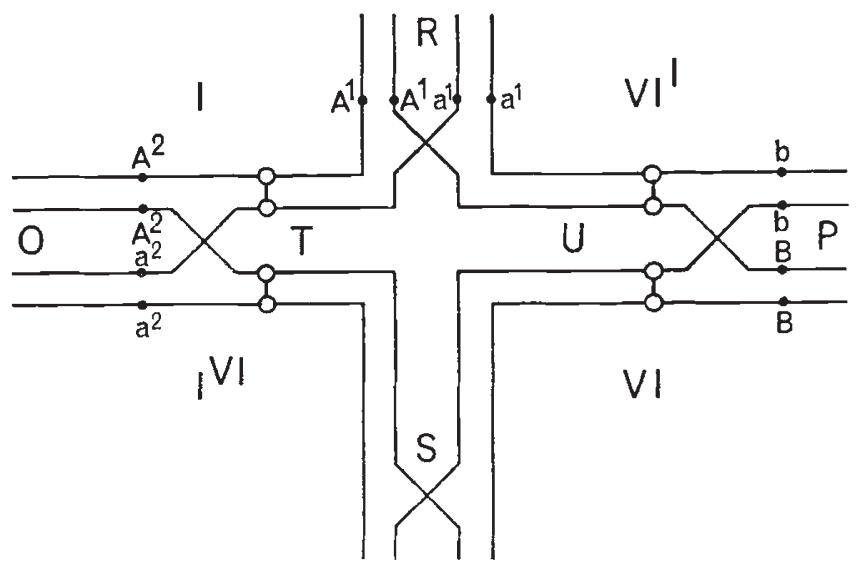

Frg. 1.-Translocation 282 pairing cross: four chromosomes (I, VI, IVI, VII $)$; six segments $(\mathrm{O}, \mathrm{P}, \mathrm{R}, \mathrm{S}, \mathrm{T}, \mathrm{U})$ and two marker genes ( $A^{\mathrm{I}}-a^{1}$ most probable location, $A^{2}-a^{2}$; alternative location, $B-b$ ). Chiasmata in $\mathrm{O}, \mathrm{P}, \mathrm{R}$ and $\mathrm{S}$; in $\mathrm{O}$ and $\mathrm{P}$ (and $\mathrm{R}$ ) between marker and centromere.

* Dedicated to Professor Dr Hans Bauer on the occasion of his 75th birthday. 
two interstitial segments ( $T$ and $U$ in fig. 1) there is usually negative interference. Between translocated and interstitial segments, however, interference is positive again. The extensive cytological evidence for this pattern has been reviewed by Sybenga (1975). Genetic evidence is scarce and old, and has not been recognised as such by the original authors. Emerson (1931) studied linkage within a translocation complex in Oenothera, and observed double crossing-over with a frequency much higher than expected. In the same report a brief note on excessive double cross-over frequencies in a Drosophila translocation heterozygote was included again without concluding that this implies negative interference. Similarly, Rhoades (1931) mentioned a coincidence value of $1 \cdot 1$ for segments opposite in respect to the breakpoint in a maize translocation heterozygote and concluded that there was "no interference".

The present report deals with an interchange heterozygote of rye. The same plants were used to analyse interference on the basis of relative metaphase I configuration frequencies, and segregations involving two marker genes and the translocation breakpoint.

\section{MAterials AND methods}

The translocation involved carries the serial number 282. It is an interchange between one of the arms of metacentric chromosome I and the long arm of submetacentric chromosome VI. These chromosome designations follow Sybenga and Wolters (1972). The correspondence with the Triticum chromosome numbering system is considered insufficiently certain to be applicable (De Vries and Sybenga, 1976). The markers used were $a$ (anthocyanin-less) on chromosome I and $b$ (brittle stem) on chromosome VI (Sybenga and Prakken, 1962). For locating these markers on the chromosomes, a telocentric trisomic for the short arm of VI was used combined with heterozygosity for $b$.

For the analysis of interference, first homozygotes for 282, $a$ and $b$ were crossed with self-fertile inbreds of the wild type. Several $\mathrm{F}_{1}$ heterozygotes were grown in a greenhouse (temperature $20-23^{\circ} \mathrm{C}$ ) during the summer of 1976. Anthers were fixed in 1:3 acetic alcohol, stored for a few months in a refrigerator at $-10^{\circ} \mathrm{C}$, and prepared for cytological analysis after aceto carmine staining. Pollen from other heads of the same plants was used to pollinate emasculated $a a b b$ plants which happened to be homozygous for translocation 282. In the segregating generations the karyotypes (trisomic and translocation respectively) were classified in root tip mitoses after pretreatment in saturated alpha bromonaphthalene for 2 hours at $24^{\circ} \mathrm{C}$; fixation-maceration in $1 \mathrm{~N} \mathrm{HCl}$ for $12 \mathrm{~min}$. at $59^{\circ} \mathrm{C}$ and Feulgen staining. Segregation for $a$ was scored at the seedling stage and for $b$ on flowering plants.

\section{RESULTS}

(i) Location of the marker genes

In the populations segregating for $b$ and the telocentric, translocation 282 segregated at the same time, but this is not relevant for the location of $b$. In the total of 76 plants without the telocentric, none carried the dominant allele $B$; in the 29 plants with the telocentric all except two were $B$. The combined segregation of telocentric and $b$ shows that the gene is located in 
the short arm of VI, fairly close to the centromere. The recombination percentage between the centromere and $b$ is affected by the pairing pattern and by the orientation of the multivalent involving the telocentric. On an average, the telocentric with $B$ will pair with one of the two normal short arms of VI (with $b$ ) in $2 / 3$ of the PMCs. With alternate orientation, a recombinant telocentric chromatid $(b)$ will always be combined with a normal chromosome with $b$, and be expressed, but with adjacent orientation in only half of the cases (as with double reduction). In rye, orientation is predominantly alternate under the conditions of the experiment, and a reduction factor of 0.9 is rather arbitrarily assumed. Then, recombinants are expected with a frequency of $p \times 2 / 3 \times 0.9$. Since there were $2 / 105$, $\mathrm{p}=3.17$ per cent. In general, recombination between the centromere and a locus is underestimated in telocentric trisomics (Endrizzi and Kohel, 1966; Sears, 1972), as a result of reduced pairing in the centromere region. Even a reduction by a factor of 4 would be possible.

There is no direct information on the location of $a$. According to Sybenga and Prakken (1962), $a$ and $b$ are not linked in normal material. In heterozygotes for translocation 282 (I-VI) $a$ and $b$ are rather closely linked (see below), whereas in heterozygotes for 240 (II-VI, with a breakpoint in VI close to that of 282), $a$ is neither linked to $b$ nor to the translocation (Sybenga and Wolters, 1972). Apparently $a$ is located in I. In homozygotes for 282, $a$ and $b$ are not linked, segregating $168: 68: 56: 12$ in an $\mathrm{F}_{2}$ of 304 .

The deviation, $\chi^{2}=4 \cdot 19(0 \cdot 1<\mathrm{P}<0 \cdot 2)$ is mainly due to shortage of $b$ and does not suggest any linkage. The preliminary conclusion is that $a$ is not in the exchanged segment, as then it would be expected to show some linkage with $b$ in the homozygote. Nor is it in an interstitial segment, as this would practically preclude recombination in the heterozygote. It is most probably situated in the non-translocation arm of $\mathrm{I}$, not far from the centromere.

\section{(ii) Interference}

(a) Cytological observations. The frequencies of the different translocation configurations in 1000 cells are given in table 1 . The analysis follows Sybenga (1975). There is a group of cells (bottom table 1) which contain only bivalents and univalents, and no multivalent translocation configuration. Since these cells are of special importance for the analysis of interference, it is necessary to estimate how many of the ring bivalents, open bivalents and univalent pairs can be attributed to the translocation complex, although these cannot be recognised microscopically. For this purpose, first the frequencies of the different bivalent configurations of normal chromosomes must be estimated in the cells in which the translocation complex can be recognised (table 2A). The bivalent and univalent distributions in the 16 cells without recognisable translocation complexes are given in table 2B. Subtracting the fraction of non-translocation bivalents and univalents from the total of table $2 \mathrm{~B}$ results in the (most probable) bivalent-univalent distribution for the translocation complex (table 2C). For instance, in table $2 \mathrm{~B}$, of the three cells of (a), with six ring bivalents and one open bivalent, most probably two contain five rings for the non-translocated chromosomes and one ring bivalent and one open bivalent for the translocation complex. The third cell would contain four ring bivalents and an open bivalent for the non-translocated chromosomes and, consequently, two ring bivalents 
TABLE 1

Frequencies of translocation configurations in $1000 \mathrm{MIPMC's} \mathrm{of} \mathrm{rye} \mathrm{plants} \mathrm{heterozygous} \mathrm{for} \mathrm{translocation}$ 282 (chromosomes $I$ and $V I$ )

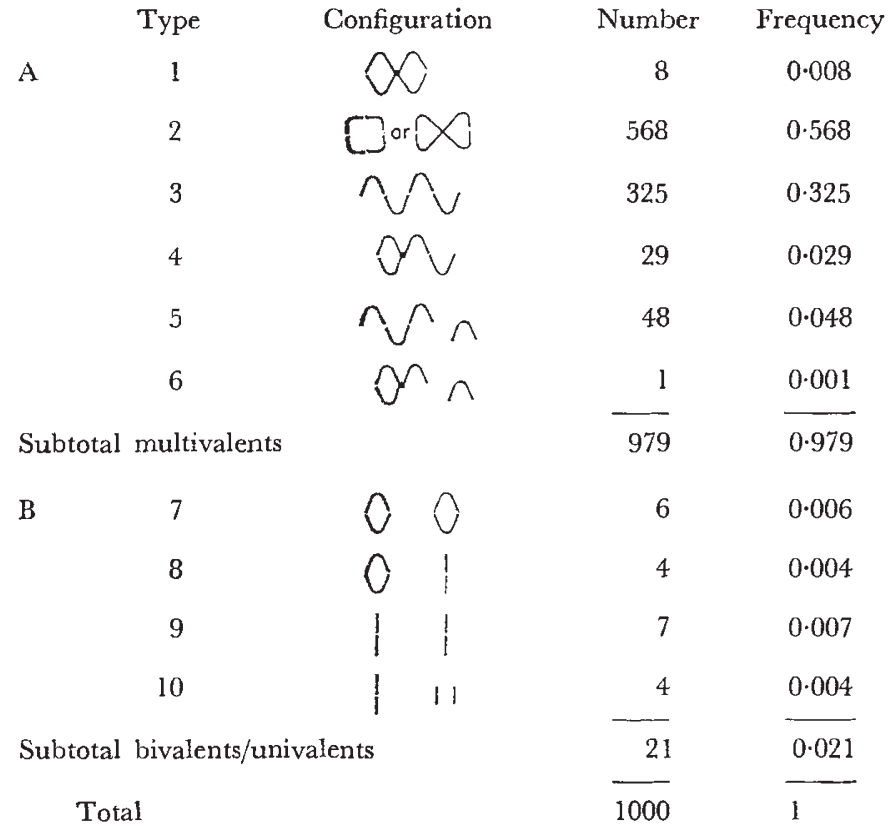

A: Multivalents. B: Bivalents and univalent pairs.

for the translocation complex. It is possible, of course, to be more " exact" and to express these frequencies properly in fractions. For our purpose, the present approach is considered satisfactory, provided possible statistical consequences are taken into account.

The analysis of crossing-over and interference in the translocation complex is split into two parts (Sybenga, 1975): one for the multivalent configuration and the other for the bivalent configurations of the translocation. The translocation pairing configuration (fig. 1) consists of six segments : $\mathrm{O}, \mathrm{P}, \mathrm{R}, \mathrm{S}, \mathrm{T}, \mathrm{U}$ with frequencies of being bound by at least one chiasma: $o, p, r, s, t, u$ respectively.

The frequencies of all configurations can be expressed in terms of $o, p, r$, etc., and these can, conversely, with the proper formulae be derived from the configuration frequencies observed. First only segments $\mathrm{O}, \mathrm{P}, \mathrm{R}$ and $\mathrm{S}$ are considered. One class of configurations (I) has all end segments bound, types 1 and 2 of table 1 ; frequency: $o \times p \times r \times s$. Type 2 requires an adjustment since it may contain configurations with $T$ and $U$ bound but not $R$ or S. Such a configuration would then belong to group II with three of the four end segments bound. Calculations show that in the present material this possibility is negligible (less than 0.4 in 1000 cells). Group II further contains types 3 and 4. Group III has two consecutive end segments bound, the other two not; types 5 and 6 . Group IV has two opposite end segments bound, and group $\mathrm{V}$ has one or no end segment bound. Now, according to Sybenga (1975) $o \times p$ is represented by $e ; r \times s$ by $f$, while $g=o+p$ and 
TABLE 2

Chromosome configurations in 1000 M I PMC's of rye plants heterozygous for translocation 282

A. Bivalent configurations in 984 cells in which the translocation complex can be recognised. Per cell five configurations besides translocation complex

$\begin{array}{cccc}\text { Rings } & \text { Open biv. } & \text { Univ. pairs } & \text { Number cells } \\ 5 & 0 & 0 & 708 \\ 4 & 1 & 0 & 256 \\ 3 & 2 & 0 & 18 \\ 2 & 3 & 0 & 1 \\ 4 & 0 & 1 & 1 \\ & & & -984\end{array}$

Total: 4919 rings $(0.94)$ and 295 open biv. $(0 \cdot 06)$.

Five cells had seven ring bivalents; two per cell must have been derived from the translocation complex, which, consequently, could be recognised.

B. Bivalent configurations in 16 cells in which the translocation complex can not be recognised.

$\begin{array}{ccccc}\text { Type code } & \text { Rings } & \text { Open biv. } & \text { Univ. pairs } & \text { Number cells } \\ \text { a } & 6 & 1 & 0 & 3 \\ \text { b } & 5 & 2 & 0 & 5 \\ \text { c } & 4 & 3 & 0 & 4 \\ \text { d } & 5 & 1 & 1 & 2 \\ \text { e } & 4 & 2 & 1 & 1 \\ \text { f } & 3 & 3 & 1 & 1 \\ & & & & 16\end{array}$

Of the 15 non-translocation bivalents of (a), on the basis of A, 0.06 or appr. 1 must be an open bivalent, the rest rings. This leaves two open bivalents in this cell type for the translocation complex, probably in different cells. In a similar way the other types can be classified. The result is given in $\mathrm{C}$.

C. Expected translocation configurations in the 16 cells of $B$, on the basis of $A$ and $B$

$\begin{array}{ccccr}\text { Type code (B) } & \text { Rings } & \text { Open biv. } & \text { Univ. pairs } & \text { Expected } \\ \text { a } & 1 & 1 & 0 & 2 \\ & 2 & 0 & 0 & 1 \\ \text { b } & 1 & 1 & 0 & 1 \\ & 0 & 2 & 0 & 4 \\ \text { c } & 1 & 1 & 0 & 1 \\ & 0 & 2 & 0 & 2 \\ \text { d } & 1 & 0 & 1 & 1 \\ \text { e } & 0 & 1 & 1 & 1 \\ \text { f } & 0 & 1 & 1 & 16\end{array}$

Combined with five cells with two rings for the translocation complex (A), this leads to the classification given in Table $\mathbf{B}$.

$\mathrm{h}=\mathrm{r}+\mathrm{s}$, and these can all be expressed in terms of $\mathrm{a}, \mathrm{b}, \mathrm{c}$ and $\mathrm{d}$, and estimated. From these expressions, o, p, r and s can be derived as the roots of two sets of quadratic equations.

In the present material it appears that $o=1.479 ; p=0.576$, while the roots for $\mathrm{r}$ and $\mathrm{s}$ have large negative discriminants. As discussed earlier (Sybenga, 1975) the latter may arise due to sampling error when the 
numbers involved are small, especially when the segments are equal in length. The size of the discriminants, however, is such that this explanation can be shown to be quite insufficient, the more so since there is no a priori reason for the segments to be equal. Negative discriminants are a convincing indication of strong negative interference between $\mathrm{R}$ and $\mathrm{S}$. It is also the cause of the absurd value for o, which, of course, is not supposed to be larger than unity. The value obtained for $p$ then is an underestimate. Since estimation of $t$ and $u$ depends on $r$ and $s$, estimates for these two cannot be derived. It is not possible, consequently, to analyse interference between $\mathrm{T}$ and $\mathrm{U}$ from the relative frequencies of multivalent configurations, according to this approach. The cells without a multivalent, although a small sample, can give more explicit indications. In the multivalents, the frequency of interstitial chiasmata (in $\mathrm{T}$ and/or $\mathrm{U}$ ) is very low : $0.008+0.030+$ $0.001=0.039$ (table 1 ). Among these, in a maximum of 0.00038 (the same frequency as used for estimating the fraction of type 2 multivalents which had to be classified in class II) both $T$ and $U$ have a chiasma. In the bivalents, however, all rings have an interstitial chiasma and perhaps even a few open bivalents (when $\mathrm{O}$ or $\mathrm{P}$ have none). Since there are several ring bivalents, 16 in 21 cells having one or more, the frequency of interstitial chiasmata here apparently is very high. This demonstrates that when there are chiasmata in $\mathrm{R}$ and /or $\mathrm{S}$, i.e., when a multivalent is formed, the probability of a chiasma in $\mathrm{T}$ and/or $\mathrm{U}$ is much smaller than when there are no chiasmata in $\mathrm{R}$ and $\mathrm{S}$, where only 0.6 would be expected among 21 cells when there would be as few as in multivalents, instead of the 16 observed. This results from positive interference between interchanged and interstitial segments and there is no reason to doubt its statistical significance.

The distribution of rings and open bivalents can be used to analyse interference between the interstitial segments $T$ and $U$. When $T$ and $U$ both have a chiasma, two rings appear. There is one ring and one open bivalent when either $\mathrm{T}$ or $\mathrm{U}$ has a chiasma. Without chiasmata in $\mathrm{T}$ and $\mathrm{U}$, there are two open bivalents. This assumes that both o and $\mathrm{p}$ equal unity, i.e., that segments $\mathrm{O}$ and $\mathrm{P}$ always have a chiasma. The estimate for $\mathrm{p}(0.576)$ suggests otherwise, but it is not reliable and grossly underestimated. It corresponds to the short arm of VI and may be assumed to be significantly smaller than 1 , perhaps about $0 \cdot 8$.

In fact, at least four of these 21 cells are estimated to have one pair of univalents (table 2). Then, the number of open bivalents given in table 1 is an overestimate and even more interstitial segments would be bound than appears from the table. This puts us on the safe side.

Now, for simplicity, assuming that o and p are equal to 1 (which underestimates interference) the 21 cells without multivalents can be grouped into three classes in respect to the association of interstitial segments: six have both associated, four have one of the two associated and 11 neither of the two.

Then, $\mathrm{tu}=\frac{6}{21}=0 \cdot 286 ; \mathrm{u}(1-\mathrm{t})+\mathrm{t}(1-\mathrm{u})=0 \cdot 190$ or $\mathrm{x}_{\mathrm{tu}}^{2}-0 \cdot 762 \mathrm{x}_{\mathrm{t}, \mathrm{u}}$ $+0.286=0$. This leads to $x_{t, u}=\frac{0 \cdot 762 \pm \sqrt{0 \cdot 762^{2}-1 \cdot 444}}{2}$ with a highly negative discriminant indicating strong negative interference between $\mathrm{T}$ and $U$ : there are far too many cells with chiasmata in both $T$ and $U$ than compatible with the number of cells with a chiasma in either $\mathrm{T}$ or $\mathrm{U}$. For 
an estimate of $\mathrm{t}+\mathrm{u}$, we have $0.039 \times 0.979$ (for the multivalents) $+16 / 21 \times$ $0.021=0.054$. Of these, 0.00638 (six in $1000+0.00038$ ) are doubles and thus, without chromatid interference, recombination in the interstitial segments amounts to $\frac{0.054-0.003}{2}=0.026$ or $2.6 \mathrm{cM}$. It can not be excluded that the group of cells lacking a translocation multivalent is a sample with relatively low average chiasma frequencies. This would result in an underestimation of the number of chiasmata in the bivalents formed by the translocation complex, and consequently lead to an even larger estimate of negative interference.

(b) Genetical observations. Table 3 gives the segregation in a test-cross of $A a B b$ TN $\times a a b b$ TT in coupling phase, and the linkage analysis. N stands for a normal genome, $\mathrm{T}$ for a translocation 282 genome. The monofactorial segregations are normal: for $a$ : $\chi^{2} 0.613(0.25<\mathrm{P}<0.5)$; for $b$ : $\chi^{2} 0.313(0.5<\mathrm{P}<0.7)$ and for $\mathrm{T}: \chi^{2} 1.013(0.25<\mathrm{P}<0.5)$.

\section{TABle 3}

Gametic segregation and linkage analysis of two genes $(\mathrm{a}, \mathrm{b})$ and translocation $282(I-V I)$ in $\mathrm{Aa} \mathrm{Bb}$ $T \mathcal{N}$, test-crossed with aa bb TT

\begin{tabular}{|c|c|c|c|}
\hline \multirow[b]{2}{*}{ Genes } & \multicolumn{2}{|c|}{ Translocation } & \multirow[b]{2}{*}{ Total } \\
\hline & $\mathrm{N}$ & $\mathrm{T}$ & \\
\hline$A B$ & 158 & 6 & 164 \\
\hline$a B$ & 0 & 1 & 1 \\
\hline$A b$ & 2 & 1 & 3 \\
\hline$a b$ & 9 & 143 & 152 \\
\hline & 169 & 151 & 320 \\
\hline
\end{tabular}

Monofactorial segregation:

$$
\begin{array}{lll}
A: a & 167: 153 & \chi^{2} 0.6125(0.50>\mathbf{P}>0.25) \\
B: b & 165: 155 & \chi^{2} 0.3125(0.7>\mathbf{P}>0.5) \\
\mathrm{N}: \mathrm{T} & 169: 151 & \chi^{2} 1.0125(0.50>\mathrm{P}>0.25)
\end{array}
$$

Recombination:

$$
\begin{array}{rlrl}
a-b & 4 / 320 & =1 \cdot 25 \% \\
a-\mathrm{T} & 16 / 320 & =5 \% \\
b-\mathrm{T} & 18 / 320 & =5 \cdot 63 \%
\end{array}
$$

Proven order $a$-T- $b$; double crossing-over $15 / 320=4 \cdot 69 \%$; expected $5 \% \times 5 \cdot 63 \%=$ $0 \cdot 28 \%$; coincidence $16 \cdot 67$. Genetic distance $a \cdot b: 34 / 320 \times 100=10 \cdot 63 \mathrm{cM}$.

The combined segregation apparently deviates from independence. Since the largest distance is $b$-T, the order of the markers would appear to be $b-a-T$. Since we know that the order is $a-T-b$, there is no reason to calculate double crossing-over and interference values for $b$ - $a$-T. Assuming the order to be $a$-T- $b$, double cross-overs ( $A$-T- $B$ and $a-\mathrm{N}-b$ ) amount to $6+9=15$ in 320 plants or 4.68 per cent Expected $16 / 320 \times 18 / 320 \times 100=0.281$ per cent. This indicates very strong negative interference, with a coincidence value of $16 \cdot 67$. Now, taking into account double crossing-over, recombination between $a$ and $b$ can be recalculated as $(21+13=34) / 320$ or 10.625 per cent. This must be an underestimate, as part of it is a result of recombination in interstitial segments, which leads to abortion of 50 per cent of the recombinant spores. Assuming that $a$ and $b$ are outside the interstitial 
segments, we have to add $0.5 \times 2.6$ per cent $=1.3$ per cent (see above), resulting in $11.9 \mathrm{cM}$ between $a$ and $b$. Most of this, apparently, is in the non-translocation arms $(9 \cdot 3$ per cent $)$.

\section{Discussion}

Since the observation of strong negative interference precludes the exchange segments as potential sites for $a$, the hypothesis that $a$ is located in the non-translocation arm of I seems to be confirmed. The segregation data then show that negative interference is not restricted to the interstitial segments but extends beyond the centromeres, only a small fraction of the total recombination taking place in the interstitial segments.

One question remains: recombination between $a$ and $b$ in the translocation heterozygote is 1.25 per cent, but the estimated crossing-over is $10 \cdot 625$ per cent. Assuming independent A II segregation of the chromatids of the translocation elements, reduction of recombination should not exceed 50 per cent (see figs. 2 and 3), i.e., 5.3 per cent recombination is the least expected, or 17 recombinants in 320 plants. This is significantly more than the four observed. There are two possible solutions:

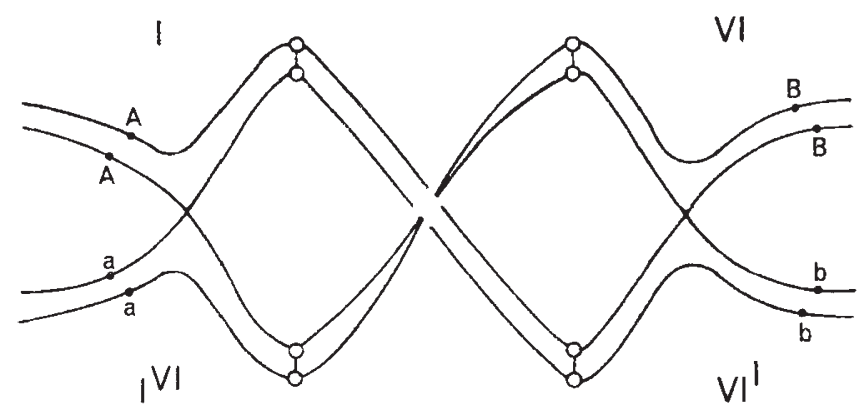

Frg. 2.-Alternate orientation of the configuration of fig. 1, resulting in "pre-orientation" for A II segregation of the centromeres by the proximal chiasmata, independent of chromatids involved.

(a) The conclusion about the location for $a$ is not correct: it is located in the translocated segment (fig. 1: $A^{1}-a^{1}$ ). The cytological data show that there is positive interference between this segment and the interstitial segment. When there is also positive interference between the interstitial segment and the proximal part of the non-translocation arm of VI (with $b$ ) this may result in negative interference between the translocated segment and the short, non-translocation arm of VI. Or, with a chiasma close to the breakpoint, recombining $a^{1}$ with the translocation breakpoint, the interstitial segment will tend to be free of chiasmata and the probability of a proximal chiasma between the centromere of VI and $b$ is large. The level of negative interference found, however, requires that the same chromatids are involved in both chiasmata: i.e., negative chromatid interference. Negative chromatid interference is less rare than usually assumed. Hearne and Huskins (1935) found it in normal chromosomes of Melanoplus and Huskins and Newcombe (1941) in Trillium. Both analysed successive chiasmata in 


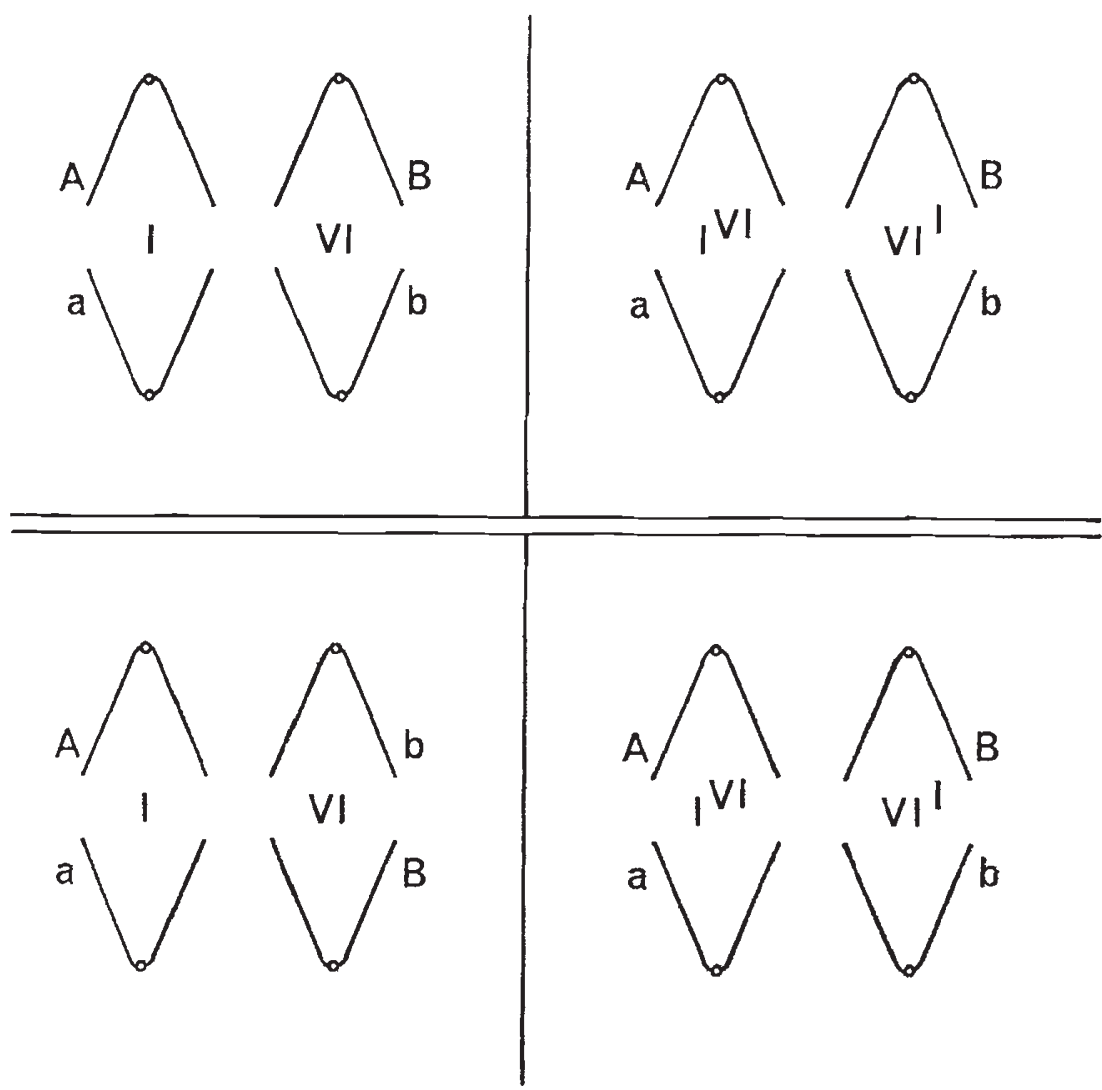

FIG. 3.-Preferential segregation for $a / b$ double cross-overs: $t o p$ : after pre-orientation by M I alternate orientation and proximal chiasmata, non-cross-over chromatids move to the same pole, as do cross-over chromatids; bottom: with independent A II segregation, even complete negative interference between $a-\mathrm{T}$ and $b-\mathrm{T}$ will not lead to lower recombination than 50 per cent of expected without negative interference.

diplotene bivalents. Correlating anaphase segregation with chiasma frequency in a Lillium callosum heteromorphic bivalent, Kayano (1959) concluded that considerable chromatid interference occurred. Comparable analysis of the translocated segment in a Disporum sessile translocation, however, demonstrated absence of chromatid interference (Kayano, 1960). Although certainly not occurring universally, negative chromatid interference is a definite possibility, cf. Sybenga (1975). Yet this explanation is improbable since the distances involved are too short to give 50 per cent recombination in the homozygote and an interference switch in the heterozygote, especially in view of the low overall recombination in rye.

(b) A proximal chiasma between centromere and genes forces the nonrecombinant chromatids to lead in A I. Combined with alternate orientation, which is predominant in rye, this results in preferential segregation in A II (figs. 2 and 3): recombinant chromatids arrive in the same spore, as do non-recombinant chromatids. Pre-orientation by anaphase I pull is well known for bridges caused by paracentric inversions in oocytes of Drosophila and EMG's of maize. Since here the segregating parent was the 
male, which does not have a linear tetrad, the process is not without complication. Yet, this explanation seems more reasonable than the previous one, in spite of the fact that we know of no previous report of such a phenomenon.

In conclusion, marker $b$ is in the short arm of chromosome VI, about $5.6 \mathrm{cM}$ from the centromere; marker $a$ is in the non-translocation arm of $\mathrm{I}$, about $5 \mathrm{cM}$ from the centromere. There is strong negative interference between the proximal segments of the non-translocated arms, reinforced by preferential segregation in A II.

Acknowledgment.- Thanks are due to Miss Anneke Stolk for analysing the combined segregation of $b$ and the telocentric.

\section{REFERENCES}

EMERSON, S. H. 1931. The inheritance of certain characters in Oenothera hybrids by different chromosome configurations. Genetics, 16, 325-348.

ENDRIZZI, J. E., AND KOHEL, R. J. 1966. Use of telosomes in mapping three chromosomes of cotton. Genetics, 54, 535-550.

HEARNE, E. M., AND HUSKINS, C. L. 1935. Chromosome pairing in Melanoplus femur-rubrum. Cytologia, 6, 123-147.

HUSKINS, C. L., AND NEWCOMBE, н. в. 1941. An analysis of chiasma pairs showing chromatid interference in Trillium erectum L. Genetics, 26, 101-127.

KAYANO, H. 1959. Chiasma studies in structural hybrids. I. Heteromorphic bivalent in Lillium callosum. Nucleus, 2, 47-50.

KAYANO, H. 1960. Chiasma studies in structural hybrids. IV. Crossing-over in Disporum sessile. Cytologia, 25, 468-475.

Newcombe, н. в. 1941. Chiasma interference in Trillium erectum. Genetics, 26, 128-136.

RHOADES, M. M. 1931. Linkage values in an interchange complex in Zea. Genetics, 17, 694-698.

SEARS, E. R. 1972. Reduced proximal crossing-over in telocentric chromosomes of wheat. Genet. Iber, 24, 233-239.

sybenGa, J. 1975. Meiotic Configurations. Springer-Verlag, Berlin, Heidelberg, New York. SYBENGA, J., AND PRAKKEN, R. 1962. Gene analysis in rye. Genetica, 33, 95-105.

SYBENGA, J., AND WOLTERS, A. H. G. 1972. The classification of the chromosomes of rye. (Secale cereale L.): a translocation tester set. Genetica, 43, 453-464.

VRIES, J. M. DE, AND SYBENGA, J. 1976. Identification of rye chromosomes: the Giemsa banding pattern and the translocation tester set. Theor. Appl. Genet., 48, 35-43. 